\title{
Performance and Degradation of Sliding Steel Friction Connections: Impact of Velocity, Corrosion Coating and Shim Material
}

\author{
G.W. Rodgers ${ }^{2}$, J.G. Chase $^{3}$, R. Causse ${ }^{1}$, J. Chanchi $^{4}$ and G.A. MacRae ${ }^{5}$
}

\section{ABSTRACT:}

It has become increasingly necessary to develop systems to decrease the impact of earthquakes by protecting people and mitigating resulting structural and economic damage. The Asymmetrical Friction Connection (AFC) or Sliding Hinge Joint (SHJ) has been intensively tested. It efficiently dissipates energy with almost no damage. However, its nonlinear mechanics have not fully been characterised.

In this study, the AFC mechanism is fully modelled and parameterised using non-linear modelling. Menegotto-Pinto models of device behavior, including added velocity dependence, are validated against a series of experimental tests. These SHJs are modelled for several shim (friction sliding surface) materials, as well as with and without corrosion resistant coatings.

The non-linear models developed accurately capture the experimentally observed nonlinear mechanics. The impact of shim material and corrosion coating on resistive force and velocity dependence are quantified. In particular, corrosion coatings create negative velocity dependence from a positive dependence without the coating. The overall modelling approach is suitable for use in a wide range of similar dynamic systems. Thus, the results also validate the overall modelling methods and the approach presented.

\section{Keywords:}

Buildings, Connections, Corrosion, Friction, Low damage, Velocity, Steel

Corresponding Author:

Associate Professor / Reader, G. A. MacRae, Gregory.macrae@canterbury.ac.nz

\footnotetext{
${ }_{1}^{1}$ Internship student, Dept. of Mechanical Engineering, University of Canterbury, Christchurch 8140, New Zealand

${ }^{2}$ Lecturer, Dept. of Mechanical Engineering, University of Canterbury, Christchurch 8140, New Zealand

${ }^{3}$ Professor, Dept. of Mechanical Engineering, University of Canterbury, Christchurch 8140, New Zealand

${ }^{4}$ PhD Student, Dept. of Civil Engineering, University of Canterbury, Christchurch 8140, New Zealand - Associate Professor at National University of Colombia, Manizales, Colombia

${ }^{5}$ Associate Professor, Dept. of Civil Engineering, University of Canterbury, Christchurch 8140, New Zealand
} 


\subsection{Introduction}

The recent series of large earthquakes that have struck New Zealand, Japan and Chile have again highlighted the potential for death, damage and downtime due to large seismic events. Significant structural damage in the beam/column connections of moment frames, can result in significant downtime and economic loss, even if the structure is reparable. In particular, the displacement caused by a large earthquake dissipates significant energy due to the sacrificial design approach used to preserve life safety, but can equally degrade structural integrity and render a structure unusable. It costs time and significant economic resource to rebuild a city after a major earthquake, and even more to regain lost prosperity of the region,. This clarifies the need for focus on emerging trends towards mitigating damage in addition to providing life safety.

Consequently, many researchers are trying to find mechanisms to increase structural life and significantly reduce economic cost by significantly decreasing damage due to seismic events. The development of damage-free methods of structural response and energy dissipation are thus at the forefront of a significant amount of research. In particular, the goal is to develop low cost connections with supplemental and repeatable mechanisms for energy dissipation that do not need replacement after an event or are easy to repair/replace. This work focuses on one such type of damage free connection, the Sliding Hinge joint (SHJ), which dissipates energy at beam-column connections through a controlled friction mechanism.

The objective of the work is the analysis, modelling and characterization of SHJ devices based on a series of experimental results [1]. It uses a well-known, fundamental mechanics based elasto-plastic model, to describe the general but nonlinear behaviour of these devices, which have a relatively high stiffness prior to sliding and kinetic friction dissipation. In particular, these devices are characterised by identifying model parameter values specific to the wide range of friction materials and corrosion coatings that can be used leading to the creation of a general model capable of capturing a wide range of SHJ connection mechanics.

Importantly, to date, there has been no full mechanics model characterisation of performance of these connections capable of capturing any given material. In addition, the impact of coating materials on dynamic or quasi-static performance has not been measured or modeled. This study addresses these issues using an existing experimental data set and develops a general model from the foundation of a well-known hysteretic 
mechanics model.

\subsection{Sliding Hinge Joint Mechanism}

The SHJ, presented below in Figure 1, is a simple device comprising a combination of plates of different materials assembled to dissipate seismic energy. It has been developed as a low-cost, efficient means to protect structures from earthquakes [2,3]. The assembly is straightforward, and friction is a function of the clamping force provided by a series of bolts and the interaction of the shim material and slotted plate. This friction mechanism enables the repeatable dissipation of seismic energy to reduce response and thus damage but keeping the structure in or near elastic domain. As a result, it provides a repeatable form of dissipation that can be used to supplement or replace sacrificial damage of the overall connection. The SHJ, sometimes also referred to as an Asymmetric Friction Connection or AFC, has been extensively tested [1-4].
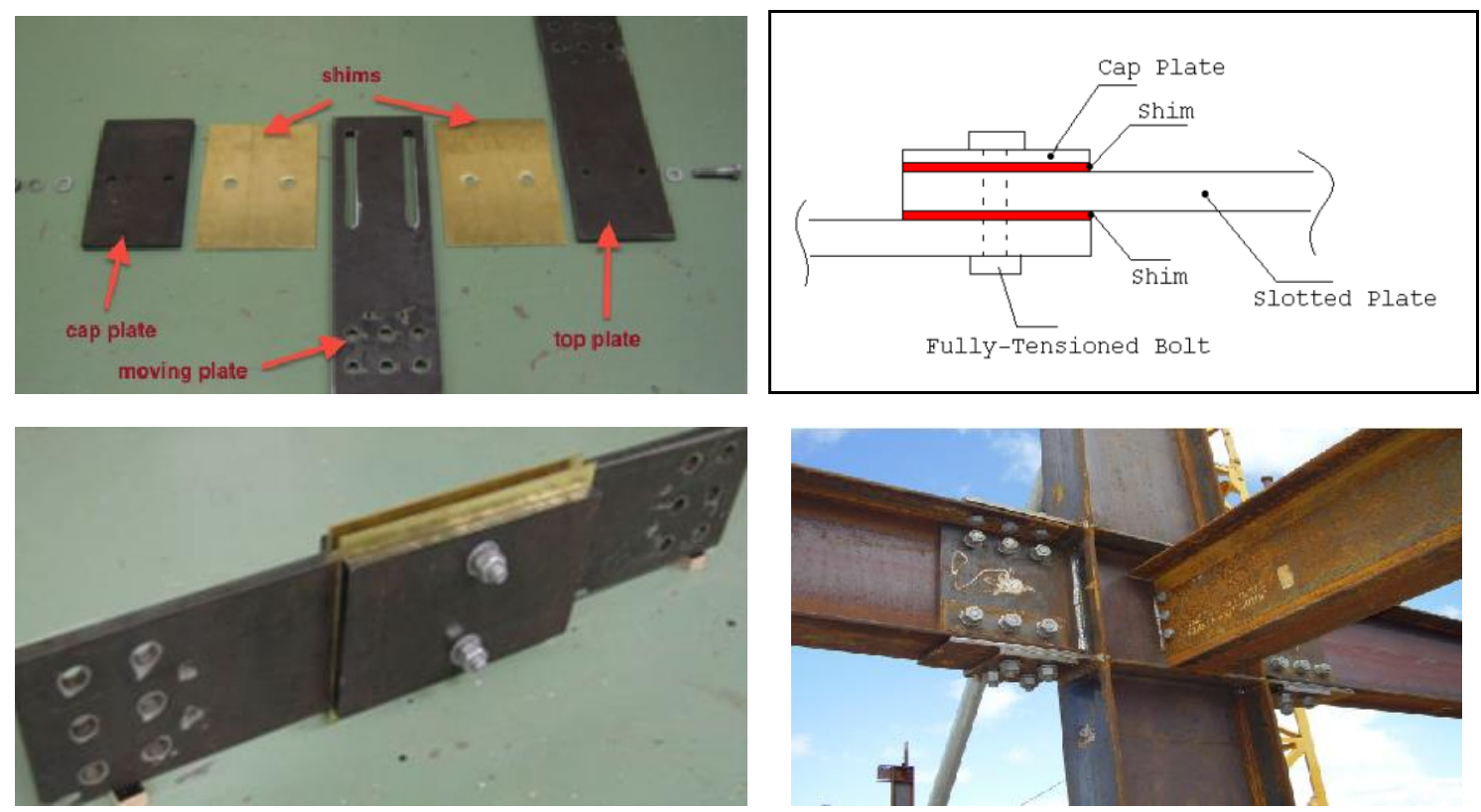

Figure 1: Sliding Hinge Joint mechanism; disassembled device (top left), two assembled views (top right and bottom left) and column/beam connection using the design approach (bottom right) [5]

More specifically, a SHJ consists of three different plates (made of steel) and two shims held together with bolts, as shown in Figure 1. The two long plates are called the moving plate and the top plate. They are drilled, enabling them to be fixed on both sides of the beams and columns to which they connect. The slots within the moving plates enable the mechanism to slide linearly when the beam and the column move. Between those three plates, shims are placed to provide friction surfaces. The movement of the moving plate 
generates friction forces in the interface between the moving plate and the shims, which propagates into the ends of the mechanism (top and cap plates). Static and kinetic friction forces are thus dependent on the shim materials used, any corrosion or other coatings, bolt torque and resulting clamping forces, and construction quality. Bolt tension is a particularly sensitive parameter in determining the resulting normal force on the shim and thus the actual device dissipation force.

\subsection{Applications of the Sliding Hinge Joint:}

Figure 2 presents different possibilities for building structures with AFC connections on single or concentrically braced frames. For single braced frames, one of the possibilities is to place the AFC at the end of the frame (Figure 2-1). It offers the advantage of being easily replaceable when damage occurs. The second alternative is to cut off the frame and fix the resulting pinned connection with the AFC (Figure 2-2). For concentrically braced frames, the AFC can be placed in two basic configurations. The AFC is directly attached to the beam bottom flange, but the two configurations differ in the arrangement of the AFC. The first solution (Figure 2-3) places the AFC in a vertical arrangement. The second configuration is a horizontal arrangement in Figure 2-4. The beam bottom flange is slotted and braces can be welded or bolted to a vertical plate welded to a horizontal plate located below the beam; two cap plates are required to be placed above the beam bottom flange. 

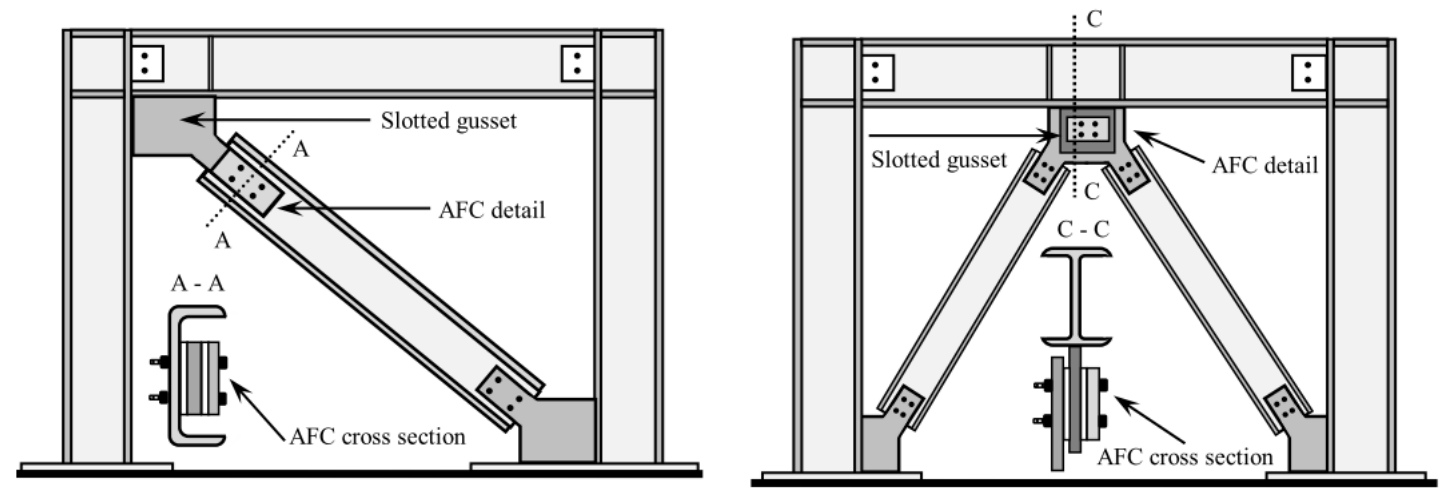

1
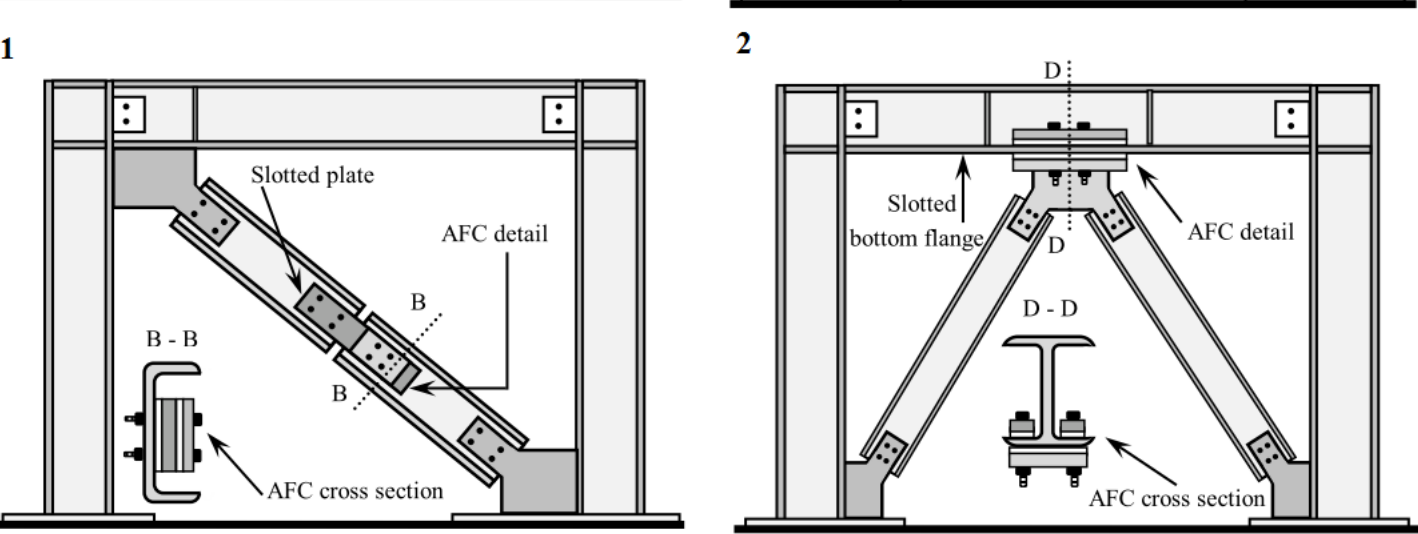

3

4

Figure 2: Applications of AFC on single and concentrically braced frames, showing single braced frames (top right and left) and concentrically frames (bottom right and left) [1]

The typical SHJ design offers the following properties $[1,4]$ :

- Long slots in the moving plate increase the displacement capacity.

- The bolt clamping force and thus the bolt torque applied control the friction force.

- Damage (if any) remains in the SHJ only and is not transmitted to the frames.

- The cost is low due to simple design and construction, and low cost materials

The SHJ is intended for structures that may be subject to high excitation, typically in regions with high seismicity. Finally, it should be note that there are several other methods and devices that can be similarly used to dissipate high energy levels in structural connections (eg [3, 6-12]). However, the focus of this work is specifically on the SHJ device and SHJ enabled steel connections. 


\subsection{Methods}

\subsection{Sliding Hinge Joint Test Input Profiles:}

This research models SHJ connections using data from experimental sinusoidal displacement inputs at several amplitudes, as friction effects can vary with length of sliding and materials. The input regimes have a constant peak sinusoidal input velocity of $10 \mathrm{~mm} / \mathrm{sec}$ to ensure a consistent evaluation of velocity dependence for each connection at any input amplitude and for any material type and coating. Comparisons are thus direct and easily made. This input velocity is based on structural simulations for use of SHJ as shown in Figure 1 at an average response velocity for a 1-2 second period, and/or when used in a typical connection.

The input displacement corresponds to six consecutive regimes of different amplitude, varying from $6.25 \%$ to $100 \%$ of the total SHJ slot length of $220 \mathrm{~mm}$. The first 5 are composed of 3 sinusoidal cycles and the last test regime has 5 input cycles, making a total of 20 cycles for each device configuration. Figure 3 and Table 1 show the input displacement details, where peak displacement corresponds to zero velocity and zero crossings are the points of maximum velocity.

To assess degradation over use, each SHJ connection was subjected to 2 of these 20 -cycle regimes. Time, displacement, and force are recorded for all tests. The displacement recorded includes both the overall test machine input motion and the direct displacement measured across the SHJ connection.

Table 1: Components of the displacement loading regime.

\begin{tabular}{|c|c|c|c|c|c|}
\hline \multirow[b]{2}{*}{ Component } & \multicolumn{2}{|c|}{ Tested Stroke } & \multirow{2}{*}{$\begin{array}{c}\text { Max. } \\
\text { Velocity } \\
(\mathrm{mm} / \mathrm{s})\end{array}$} & \multirow{2}{*}{$\begin{array}{l}\text { Frequency } \\
(\mathrm{Hz})\end{array}$} & \multirow{2}{*}{$\begin{array}{c}\text { Cycles } \\
(\#)\end{array}$} \\
\hline & $(\%)$ & $(\mathrm{mm})$ & & & \\
\hline 1 & 6.25 & \pm 6 & 10 & 0.27 & 3 \\
\hline 2 & 12.5 & \pm 12 & 10 & 0.13 & 3 \\
\hline 3 & 25 & \pm 24 & 10 & 0.067 & 3 \\
\hline 4 & 50 & \pm 48 & 10 & 0.034 & 3 \\
\hline 5 & 75 & \pm 72 & 10 & 0.022 & 3 \\
\hline 6 & 100 & \pm 95 & 10 & 0.017 & 6 \\
\hline
\end{tabular}




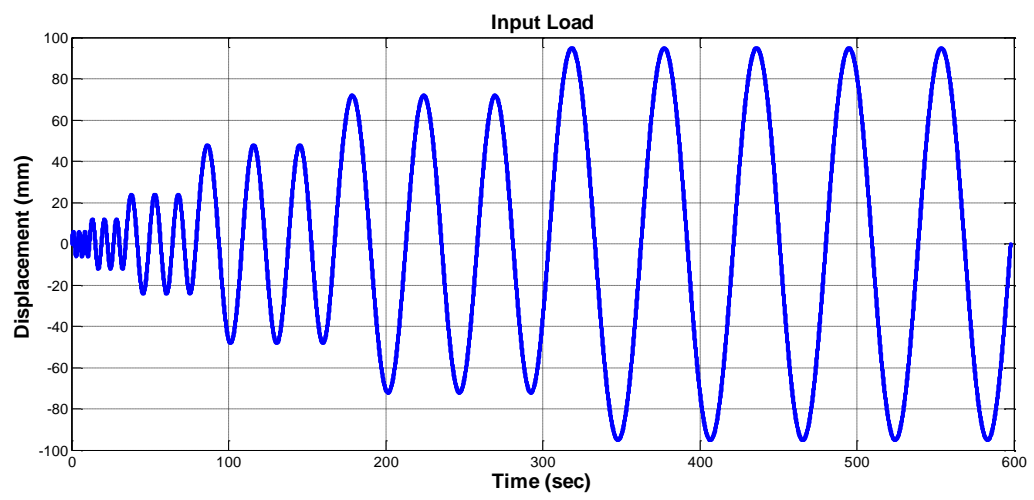

Figure 3: Loading Regime: Input displacement (mm) over time (s)

\subsection{Shim Materials and SHJ Construction:}

Six shim materials listed in Table 2 were tested to quantify their impact on friction force and the resulting device hysteresis loop. Each connection used two M16 Grade 8.8 bolts with a $220 \mathrm{~mm}$ slot length, and a 3$6 \mathrm{~mm}$ thick shim. The cap, moving and fixed plates of the SHJ are $20 \mathrm{~mm}$ thick steel. Bolts were consistently tensioned to $390 / 400 \mathrm{kN}$, validated as the bolt proof load, to ensure equal comparisons and reflect a standard form that would yield consistent assembly.

Table 2: Details of the different shim materials used in the SHJ connections

\begin{tabular}{|c|c|c|}
\cline { 2 - 3 } \multicolumn{1}{c|}{} & Material & Specification \\
\hline \multirow{5}{*}{$\frac{\text { Aluminium }}{\bar{\omega}}$} & Brass & 5005Gp-Series Aluminium \\
& Bisalloy 80 & BNS $26000-1 / 2$ Hard Temper \\
& Bisalloy 400 & Bisplate 80 \\
& Bisalloy 500 & Bisplate 500 \\
\hline
\end{tabular}

\subsection{Sliding Hinge Joint Anti-Corrosion Coatings:}

Corrosion resistance is important in steel structures and devices may be exposed to a moisture and corrosive environment. A second set of tests examines anti-corrosion coatings. Five anti-corrosion coatings are tested using a $6 \mathrm{~mm}$ Bisalloy 500 shim material for consistency to quantify the impact, if any, of anti-corrosion coatings on the resulting sliding friction and device forces. The specific coatings tested include: 1) Sweep blasted; 2) alkyd $70 \%$; 3) alkyd 100\%; 4) zinc70\%; and 5) zinc 100\%. The percentages are the amount of the sliding surface covered and are based on typical uses. 


\subsection{Corrosion Tests:}

Finally, the original SHJ connections using a Bisalloy 500 shim and each type of corrosion coating are subjected to $24 \times 6$-hour cycles of soaking in salted water, each followed by 18 hours of drying. The tests were carried out with water of $40-50^{\circ} \mathrm{C}$ and $36 \mathrm{~g} / \mathrm{L}$ of salt concentration. Devices were then further tested to quantify the impact of this corrosive environment on the ability to generate friction forces and quantify any degradation or other change.

\subsection{Sliding Hinge Joint Modelling:}

Figure 4 presents an example experimental hysteresis loop. The goal in this work is to model these loops with a fundamental mechanics elasto-plastic model. The Menegotto-Pinto model is an effective method of simulating the observed elasto-plastic behaviour of the SHJ connection, where the connection force is a function of input displacement. However, the slight rise in force at peak velocity crossing zero displacement indicates some measure of velocity dependence that is not expected in these devices [4].

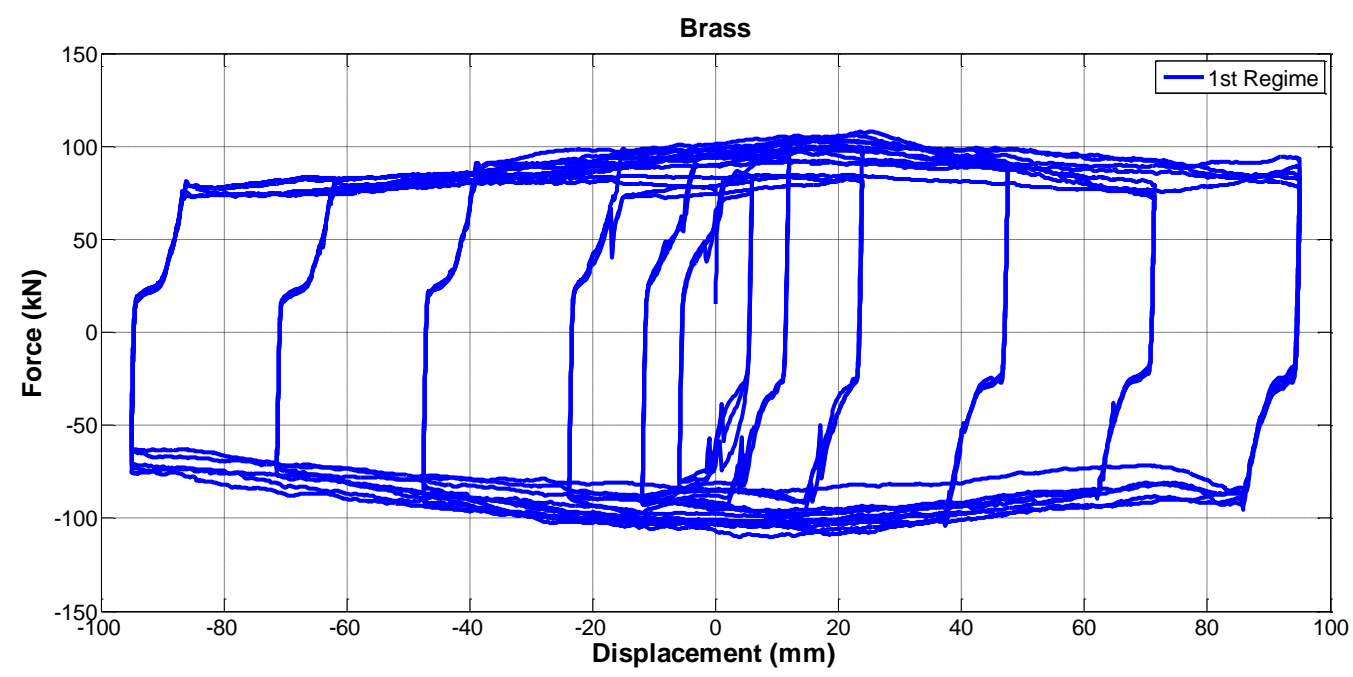

Figure 4: Experimental hysteresis loop for brass shims and a sinusoidal input of Figure 3; Force $(\mathrm{kN})$ over displacement $(\mathrm{mm})$, from [1].

Equation (1) presents the fundamental description of the Menegotto-Pinto model [13]:

$$
F=\frac{K \cdot\left(x-x_{\text {reset }}\right)+F_{\text {reset }}}{\left(1+\left|\frac{K \cdot\left(x-x_{\text {reset }}\right)+F_{\text {reset }}}{F_{y} \cdot(\operatorname{sign}(\dot{x}))}\right|^{\beta}\right)^{1 / \beta}}
$$

Where $F_{y}$ is the sliding force threshold, $K$ is the initial linear stiffness, $X_{\text {reset }}$ is the displacement at the last time 
the velocity changed sign, $F_{\text {reset }}$ is the force at the last time that the velocity changed sign, and $\beta$ is a constant that determines the rate of divergence. For high values of $\beta$ the elastic-plastic transition is very sharp, whereas lower values of $\beta$ give a smoother, more gradual transition. All these terms are defined schematically in Figure 5.
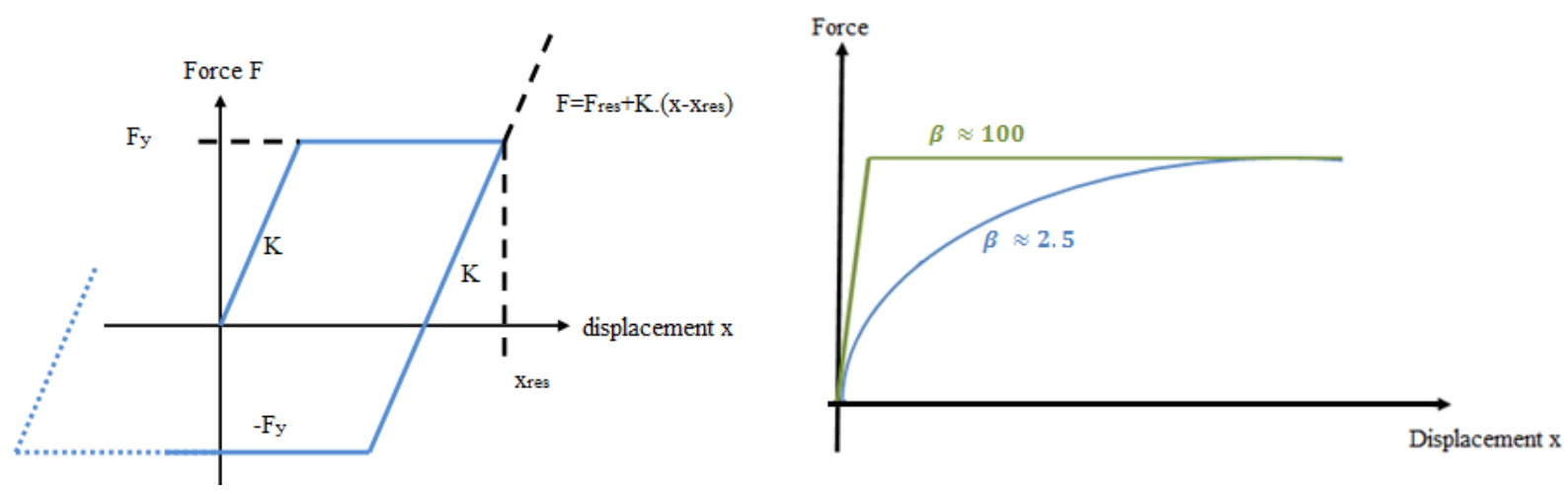

Figure 5: Hysteresis loop parameter definitions the perfect elasto-plastic Menegotto-Pinto model.

Each experiment is modelled and all model parameters identified for each case. A specific added effect included in this work is the addition of velocity dependence, evident in Figure 4 at zero displacement crossings and thus peak velocity of the sinusoidal input. This velocity dependence finding is unique to this work, as the SHJ was previously considered to be velocity independent [1, 2, 4], but is based on rigorous experimental results. The model including velocity dependence as a linear term is thus defined:

$$
F_{\text {new model }}=F_{\text {Menegotto }- \text { Pinto }} \cdot(1+v / \text { dep })
$$

Where $F_{\text {Menegotto-Pinto }}$ is the force calculated with the Menegotto-Pinto model in Equation (1), $v$ is the velocity and $d e p$ the velocity dependence factor, both with units of $\mathrm{mm} / \mathrm{sec}$ creating a unit-less ratio. The unit-less multiplicative term $(1+v / d e p)$ thus captures the static force and added force due to velocity dependence.

\subsection{Summary of Analyses:}

This unique data set enables the quantification for the first time of SHJ device force and velocity dependence as a function of: 
- Shim material (Aluminium, Brass, Bisalloy 80, Bisalloy 400, Bisalloy 500)

- Corrosion coating and coverage (Sweep Blasted, Alkyd, Zinc on a Bisalloy 500 shim)

- Prior large input cycles (Across 2 input cycle regimes)

- Corrosion (Salt water bath exposure)

The results are used to quantify these factors and capture their behaviour in a single device model suitable for use in design. 


\subsection{Results and Discussion}

\subsection{Velocity Dependence and Initial Tests:}

Figure 6 presents the hysteresis loop of a SHJ using brass shims, with and without identified model velocity dependence terms, for both 20 cycle test regimes. Both panels show experimental and identified model results, showing a good overall match. The vertical line at every switch of direction is due to experimental error where this device slid rigidly (plate and shim) back to zero and is a construction and experimental error, rather than a standard device response. More importantly and unique to these results, the need for velocity dependence is clear due to the mismatch of the experimental and model results in the top row. The velocity dependence enables the curve of the top and bottom of the loop to be more accurately captured.
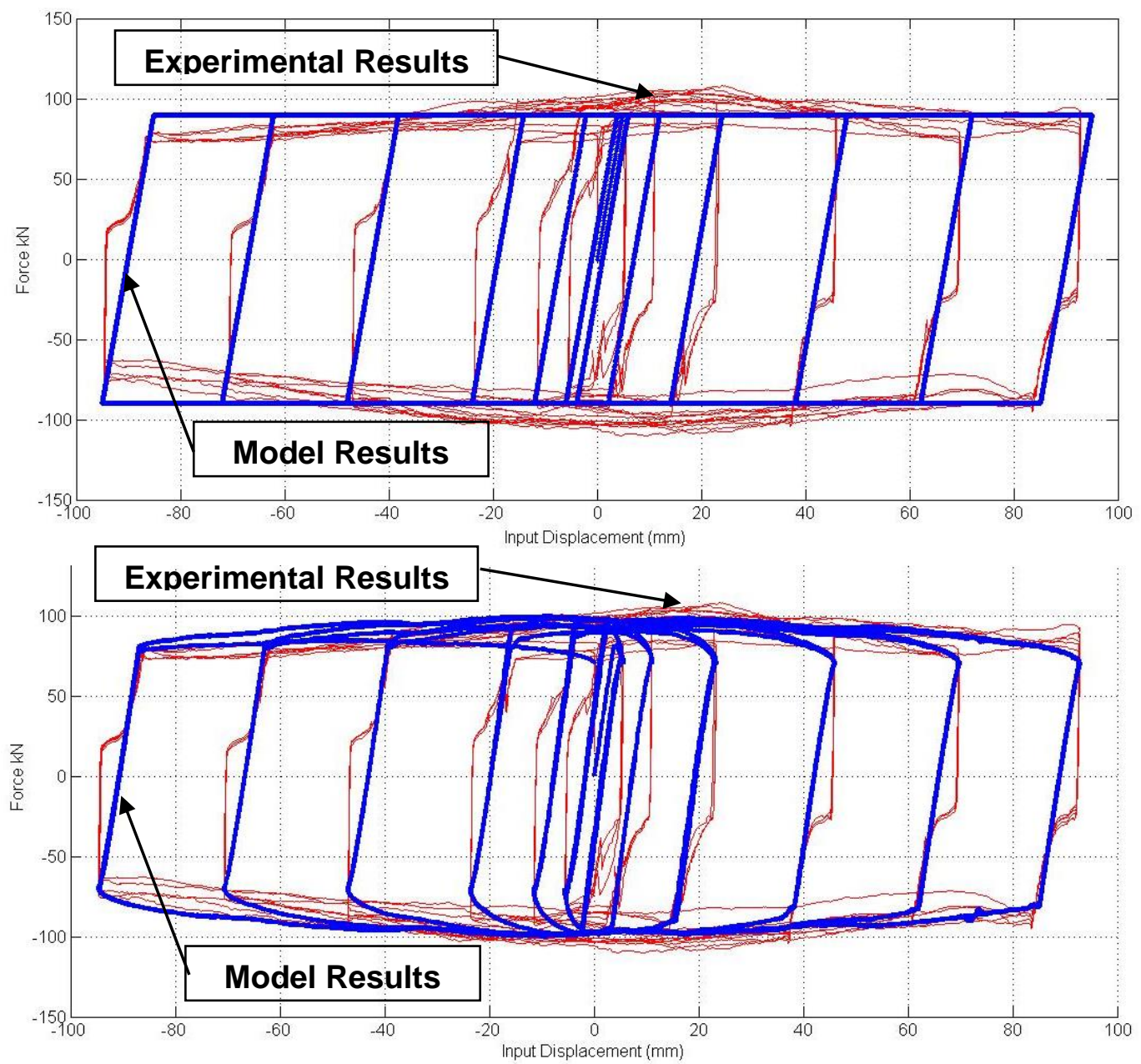

Figure 6: Hysteresis loop for the brass shim. The top panel shows experimental (single thick line in blue) and model results (multiple thin lines in red) without velocity dependence, while the bottom presents the same experimental results with model results including velocity dependence. Model and experimental results are also labeled in the figures directly. 
Table 3 summarises the model parameters for all shim materials and test regimes. The stiffness, the rate of divergence $\beta$ and the dependence parameter do not change between the 1 st and 2 nd regime. The only parameter varying is the sliding force $F_{y}$, which changes due to degradation of the devices due to prior input cycles and the resulting loss of clamping force, which has been observed previously [1]. The quality of fit is very similar to those in Figure 6 (not shown) indicating the overall model structure captures the observed mechanics and behaviour.

The velocity dependence is significant, but does not change between regimes, even though the overall force does. However, it is clear from the results and Figure 6 that is necessary to capture all the observed force behaviour. In particular, the velocity dependence term, dep, in Table 3 , can vary significantly between materials, indicating the different friction properties of these materials. Finally, the fact that it does not change between test regime runs indicates it is a function of the material and not affected, in this model formulation, by the bolt clamping forces or other changes, which should help further validate the overall model. Overall, this velocity dependence is one unique finding of this work, where previously it was thought that Coulomb friction devices would not display a velocity dependence effect [1, 2, 4].

Table 3: Model parameters for each shim material

\begin{tabular}{|c|c|c|c|c|c|c|c|c|}
\hline \multirow{2}{*}{ sin $e^{e^{x s}}$} & \multicolumn{2}{|c|}{ Stiffness K (kN.m-1) } & \multicolumn{2}{|c|}{ Sliding force Fy (kN) } & \multicolumn{2}{|c|}{ Rate of divergence $\beta$} & \multicolumn{2}{|c|}{ Dep } \\
\hline & 1st Run & 2nd Run & 1st Run & 2nd Run & 1st Run & 2nd Run & 1st Run & 2nd Run \\
\hline Brass & 18 & 18 & 70 & 50 & 100 & 100 & 30 & 30 \\
\hline Aluminium & 17.5 & 17.5 & 50 & 45 & 100 & 100 & 60 & 60 \\
\hline Bisalloy 80 & 20 & 20 & 70 & 50 & 100 & 100 & 60 & 60 \\
\hline Bisalloy 400 & 22 & 22 & 65 & 60 & 100 & 100 & 30 & 30 \\
\hline Bisalloy 500 & 18 & 18 & 80 & 70 & 100 & 100 & 60 & 60 \\
\hline
\end{tabular}

However, equally importantly, there are degradations in sliding force between regimes. The $2^{\text {nd }}$ Run results for Sliding Force, $F_{y}$, are $10-30 \%$ lower than those of the first run in this case. In contrast, all other parameters remain constant between runs, as might be expected. Hence, the peak forces and model generated hysteresis loops would be as in Figure 6, but scaled, along with the experimental data, validating that only the effective yield force is changed by prior exposure to large input cycles.

More importantly, this degradation should be noted because subsequent large aftershocks would see similar 
reductions in energy dissipation or larger. In these experimental tests, as described here and in [1], the input was from a shake table. The plates either side or the dissipation zone were held horizontal, so the possibility of out-of-plane prying deformations that can occur in real connections were held to zero or near zero. Prying occurs when the input to the three bolted plates is not perfectly horizontal. Out of plane motions, even small ones, act to reduce the tension of the bolts on the plates, which in turn reduce the total friction forces, and thus the device forces and dissipation. In AFC connections in more realistic applications, prying deformations are more likely to occur, reducing bolt clamping forces and thus device friction and dissipation. Thus, the results presented here are the minimum degradation expected since prying was eliminated, noting that prying effects and resulting reductions in device forces can be large $[14,15]$.

\subsection{Impact of Anti-Corrosion Coatings:}

Figure 7 shows the hysteresis loop of the SHJ with Bisalloy 500 shim with a sweep blasted anti-corrosion coating. The Menegotto-Pinto model fits the experimental results well. However, the negative velocity dependence parameter required is in stark contrast to results for uncoated devices in Figure 6 and Table 3 , which have positive coefficients. The negative velocity coefficient is required to capture the reduced force observed experimentally at peak sinusoidal input velocity as the device position passes zero. It is clear that this particular anti-corrosion coating appears to act as a form of lubricant under this larger cyclic loading, degrading the level of resistive force at peak velocity around the zero deflection point. More importantly, this negative velocity coefficient and dependence was required for all coatings tested, as seen in Table 4, except for the Alkyd 100 coating also shown in Figure 8. More specifically, it was experimentally observed after testing that coated devices left a faint powder from degraded coating material removed under friction, and it is thus hypothesized that these particles effectively act as a lubricant to motion, reducing friction at higher velocity in the sinusoidal input and thus requiring a negative velocity coefficient.
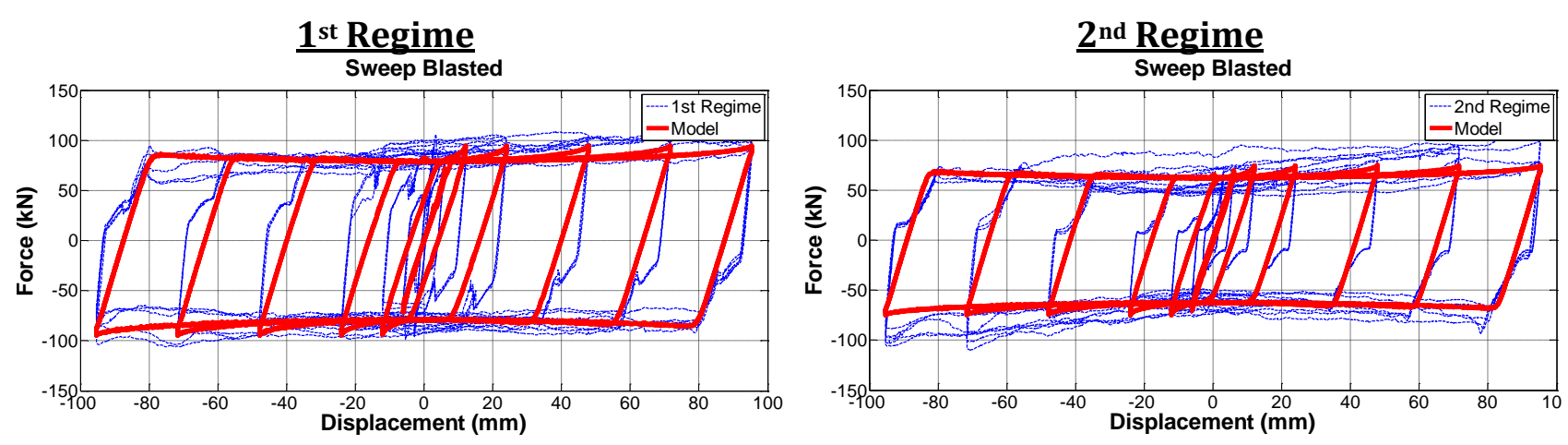

Figure 7: Control coating tests: sweep blasted hysteresis loop, including the velocity dependence term, for both sets of test input regimes. 
The overall results are summarised in Table 4 for both the $1^{\text {st }}$ and $2^{\text {nd }}$ test regime. Note that Table 4 also shows a comparison to the uncoated results for Bisalloy 500 in Table 3 for direct comparison and context. As in Table 3, the results are consistent between test regimes, except for the device force, which evidences degradation due to the prior inputs, and is likely due to the loss of bolt clamping forces. Hence, these results further validate the model structure and approach chosen.
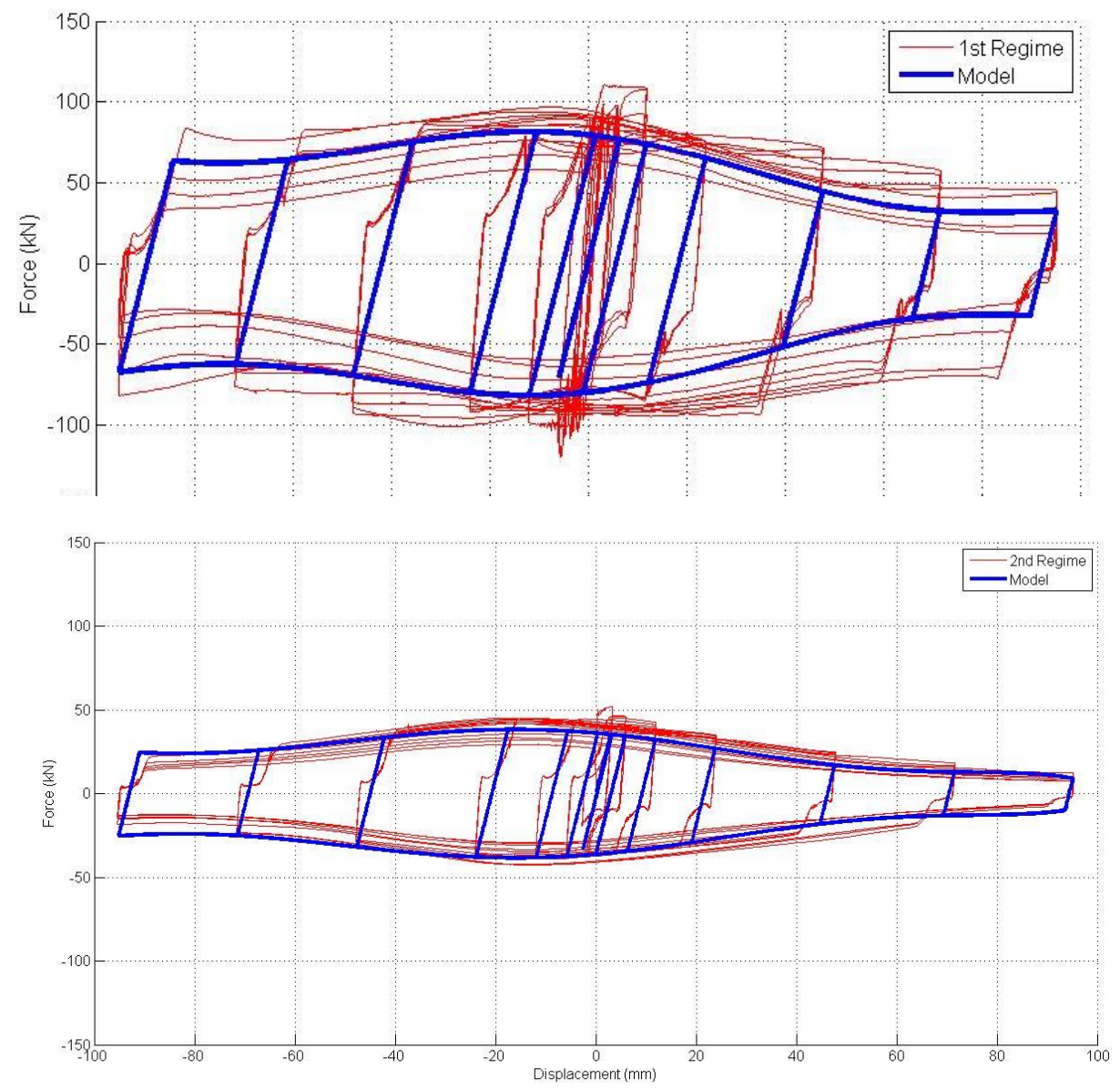

Figure 8: Alkyd 100 test and model results for Regime 1 (top) and Regime 2 (bottom), showing the clear degradation in force (same axes), as well as positive velocity coefficient in Tables 4-5.

However, the degradation is greater than that observed in Table 3 for uncoated devices, and repeated in Table 4, for the uncoated Bisalloy 500 case. In particular, degradation up to $\sim 60 \%$ can be observed, where this worst case is for the Alkyd 100 coating, which is also shown in Figure 8. There is thus an increased degradation or loss of force due to the form of lubrication that some of the anti-corrosion coatings provide, as that is the only variable that has changed from Table 3. Importantly, a second set of tests on identical devices, with results shown in Table 5, confirmed this unexpected result and further validated the results and 
model.

An equally interesting outcome of this repetition is seen in the slight differences between Tables 4 and 5 . These differences between otherwise identical devices are attributed to smaller differences in construction and materials from device to device. These differences thus also show how difficult it might be in practice to achieve relatively identical results in fabrication and construction. Hence, this second set of tests, constructed only to confirm the unexpected results in Table 4, also provide insight into device to device variability due to small differences in materials and/or fabrication.

Table 4: Model parameters for each anti-corrosion coating: $1^{\text {st }}$ and $2^{\text {nd }}$ test regime results.

\begin{tabular}{|c|c|c|c|c|c|c|c|c|}
\hline \multirow{2}{*}{ 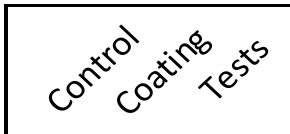 } & \multicolumn{2}{|c|}{ K } & \multicolumn{2}{|c|}{ Fy } & \multicolumn{2}{|c|}{ B } & \multicolumn{2}{|c|}{ Dep } \\
\hline & 1st Run & 2nd Run & 1st Run & 2nd Run & 1st Run & 2nd Run & 1st Run & 2nd Run \\
\hline Sweep Blasted & 12 & 12 & 95 & 75 & 10 & 10 & -90 & -90 \\
\hline Alkyd 70\% & 15 & 15 & 90 & 78 & 10 & 10 & -30 & -30 \\
\hline Alkyd $100 \%$ & 8 & 8 & 50 & 20 & 10 & 10 & 30 & 30 \\
\hline Zinc 70\% & 10 & 10 & 90 & 60 & 10 & 10 & -90 & -90 \\
\hline Zinc $100 \%$ & 10 & 10 & 90 & 60 & 10 & 10 & -90 & -90 \\
\hline $\begin{array}{c}\text { Uncoated results } \\
\text { Bisalloy } 500\end{array}$ & 18 & 18 & 80 & 70 & 100 & 100 & 60 & 60 \\
\hline
\end{tabular}

Table 5: Model parameters for each coating: a second set of results on identical devices to confirm the results of Table 4.

\begin{tabular}{|c|c|c|c|c|c|c|c|c|}
\hline 0 & \multicolumn{2}{|c|}{ K } & \multicolumn{2}{|c|}{ Fy } & \multicolumn{2}{|c|}{ B } & \multicolumn{2}{|c|}{ Dep } \\
\hline $\mathrm{C}^{\circ} \mathrm{C}^{\circ}$ & 1st Run & 2nd Run & 1st Run & 2nd Run & 1st Run & 2nd Run & 1st Run & 2nd Run \\
\hline Sweep Blasted & 14 & 14 & 95 & 75 & 10 & 10 & -120 & -120 \\
\hline Alkyd 70\% & 15 & 15 & 90 & 78 & 10 & 10 & -30 & -30 \\
\hline Alkyd $100 \%$ & 12 & 12 & 75 & 45 & 10 & 10 & -60 & -60 \\
\hline Zinc $70 \%$ & 13 & 13 & 90 & 70 & 10 & 10 & -60 & -60 \\
\hline Zinc $100 \%$ & 10 & 10 & 50 & 10 & 10 & 10 & -100 & -100 \\
\hline $\begin{array}{c}\text { Uncoated results } \\
\text { Bisalloy } 500\end{array}$ & 18 & 18 & 80 & 70 & 100 & 100 & 60 & 60 \\
\hline
\end{tabular}

Finally, Figure 8 also shows that the effect of anti-corrosion coating under friction can add some variability to the hysteresis loop. Compared to Figure 6, or even Figure 7, the results in Figure 8 show greater variability from the model, as well as from expected, relatively square Coulomb friction hysteresis loops [2, 4]. Such differences between expected and outcome behaviours are a further unique outcome of this work. They could also prove significant in use, but, as seen in the modeled versus experimental results, can now be 
accounted for in design.

\subsection{Impact of Corrosion:}

Figure 9 shows the hysteresis loop for a sweep blasted coating after corrosion testing. The Menegotto-Pinto model fits the experimental results well, again requiring a negative velocity dependence parameter to capture the observed mechanics. It is similar in shape to Figure 7, for an identical connection, as expected, and further confirming the efficacy of this augmented Menegotto-Pinto model formulation for capturing the observed mechanics of these devices.

Tables 6-7 summarize the results for the two sets of devices available for corrosion testing, and represent those in Tables 4 and 5 , respectively. Overall, comparing Tables $4-5$ to Tables $6-7$, it is clear that the impact of corrosion, as tested, was limited. In particular, corrosion testing had no notable effect outside expected variation observed between devices using the same materials, which is an encouraging result for the longterm use of these devices.
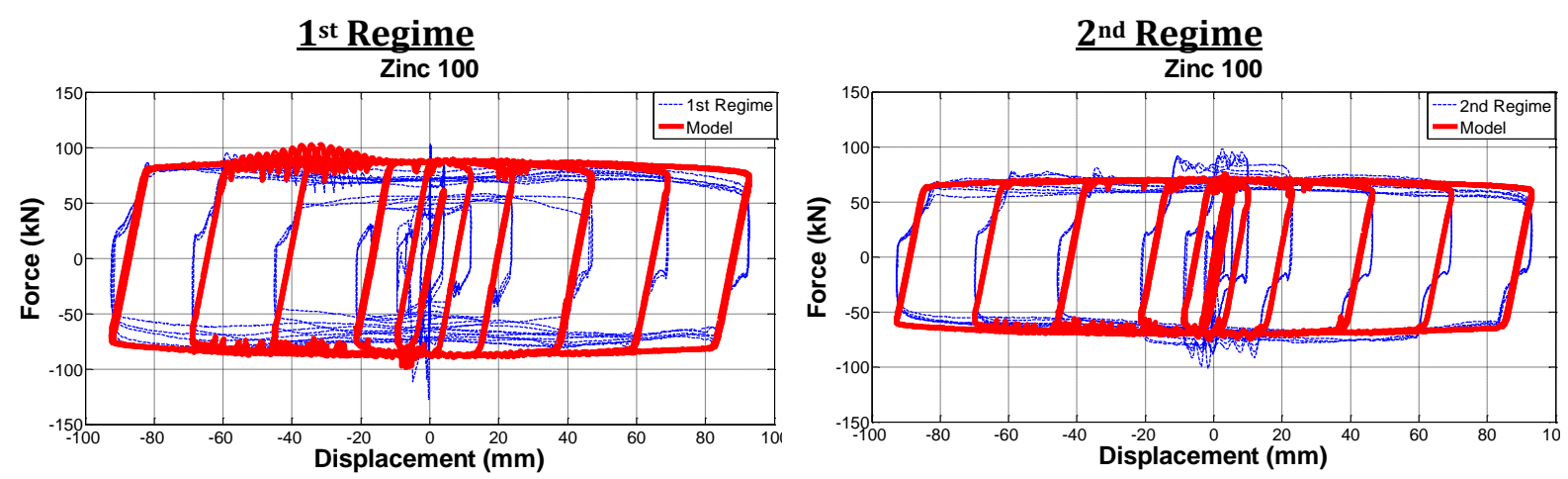

Figure 9: Corrosion tests: Hysteresis loops for sweep blasted coating, both test regimes.

Table 6: Model parameters for each coating corroded: first set of results

\begin{tabular}{|c|c|c|c|c|c|c|c|c|}
\hline or stratrats & \multicolumn{2}{|c|}{$\bar{K}$} & \multicolumn{2}{|c|}{ Fy } & \multicolumn{2}{|c|}{ B } & \multicolumn{2}{|c|}{ Dep } \\
\hline $0^{x}$ & 1st Run & 2nd Run & 1st Run & 2nd Run & 1st Run & 2nd Run & 1st Run & 2nd Run \\
\hline Sweep Blasted & 12 & 12 & 65 & 60 & 10 & 10 & -60 & -60 \\
\hline Alkyd 70\% & 12 & 12 & 55 & 38 & 10 & 10 & -60 & -60 \\
\hline Alkyd 100\% & 18 & 18 & 85 & 45 & 10 & 10 & -150 & -150 \\
\hline Zinc 70\% & 18 & 18 & 72 & 65 & 10 & 10 & 90 & 90 \\
\hline Zinc $100 \%$ & 15 & 15 & 75 & 60 & 10 & 10 & 90 & 90 \\
\hline
\end{tabular}


Table 7: Model parameters for each coating corroded: second set of results.

\begin{tabular}{|c|c|c|c|c|c|c|c|c|}
\hline & \multicolumn{2}{|c|}{$\mathrm{K}$} & \multicolumn{2}{|c|}{ Fy } & \multicolumn{2}{|c|}{ B } & \multicolumn{2}{|c|}{ Dep } \\
\hline $\mathrm{cos}^{\prime \prime}$ & 1st Run & 2nd Run & 1st Run & 2nd Run & 1st Run & 2nd Run & 1st Run & 2nd Run \\
\hline Sweep Blasted & 15 & 15 & 60 & 50 & 10 & 10 & -60 & -60 \\
\hline Alkyd 70\% & 12 & 12 & 55 & 38 & 10 & 10 & -60 & -60 \\
\hline Alkyd $100 \%$ & 6 & 6 & 22 & 16 & 10 & 10 & -150 & -150 \\
\hline Zinc $70 \%$ & 18 & 18 & 72 & 65 & 10 & 10 & -90 & -90 \\
\hline Zinc $100 \%$ & 15 & 15 & 75 & 45 & 10 & 10 & -90 & -90 \\
\hline
\end{tabular}

\subsection{Overall Results:}

Velocity dependence is a unique finding of this work and a unique aspect of the augmented model developed to capture this effect. Figure 9 summarizes the overall velocity dependence results for a Bisalloy 500 shim SHJ. The error bars in Figure 9 show the variation of the results. As shown, the two last values are equal to zero. However, this value does not mean that no dependence has been found. In fact, the velocity dependence was either positive or negative with the same intensity. Moreover, the bars represent the intensity average of the velocity dependence. The closer the value is to zero, the more important the velocity dependence is. Therefore, a specific percentage would not be very meaningful and it is more useful to note only the positive/negative velocity dependence. Importantly, overall, the coating and corrosion results yield an unexpected negative velocity dependence parameter, except for the non-coated and non-corroded SHJ connections.

Force degradation was also different between materials and coatings. Figure 10 summarises the force degradation between test regimes as a percentage of the original, $1^{\text {st }}$ test regime results. In particular, most cases do not exceed $30 \%$ of degradation between the $1^{\text {st }}$ and $2^{\text {nd }}$ run. In particular, 6 have a degradation of $10 \%$ and 5 of $30 \%$. However, 2 tests of the coating trial show that the degradation can reach $50-60 \%$. Overall, degradation was greater for the anti-corrosion coated devices than for the untreated devices, which is attributed to the lubricating effect that friction on these coatings yields and that is evidenced in the negative velocity dependence coefficients and these results.

Finally, Figure 11 shows a wide range of experimental results for overall context and to qualitatively highlight the variability across devices, materials and coatings in hysteretic behaviour. Importantly, the level of velocity dependence, from positive to negative values, is also highly variable. These results are solely presented for qualitative comparison to illustrate the variability observed. Equally, they show that the Menegotto-pinto model 
with velocity dependence is able to replicate the fundamental behaviours of each connection, regardless of material type or coating, as well as before and after large input cycles where degradation may occur. Both the oscillations and the curved plateau of the hysteresis loop are well captured in each case with no outlying differences between model and experimental results, providing a good overall qualitative model assessment across this range. The very high noise in the top right panel for the Zinc $100 \%$ coating is due to a unique stick-slip phenomenon in these tests [16].

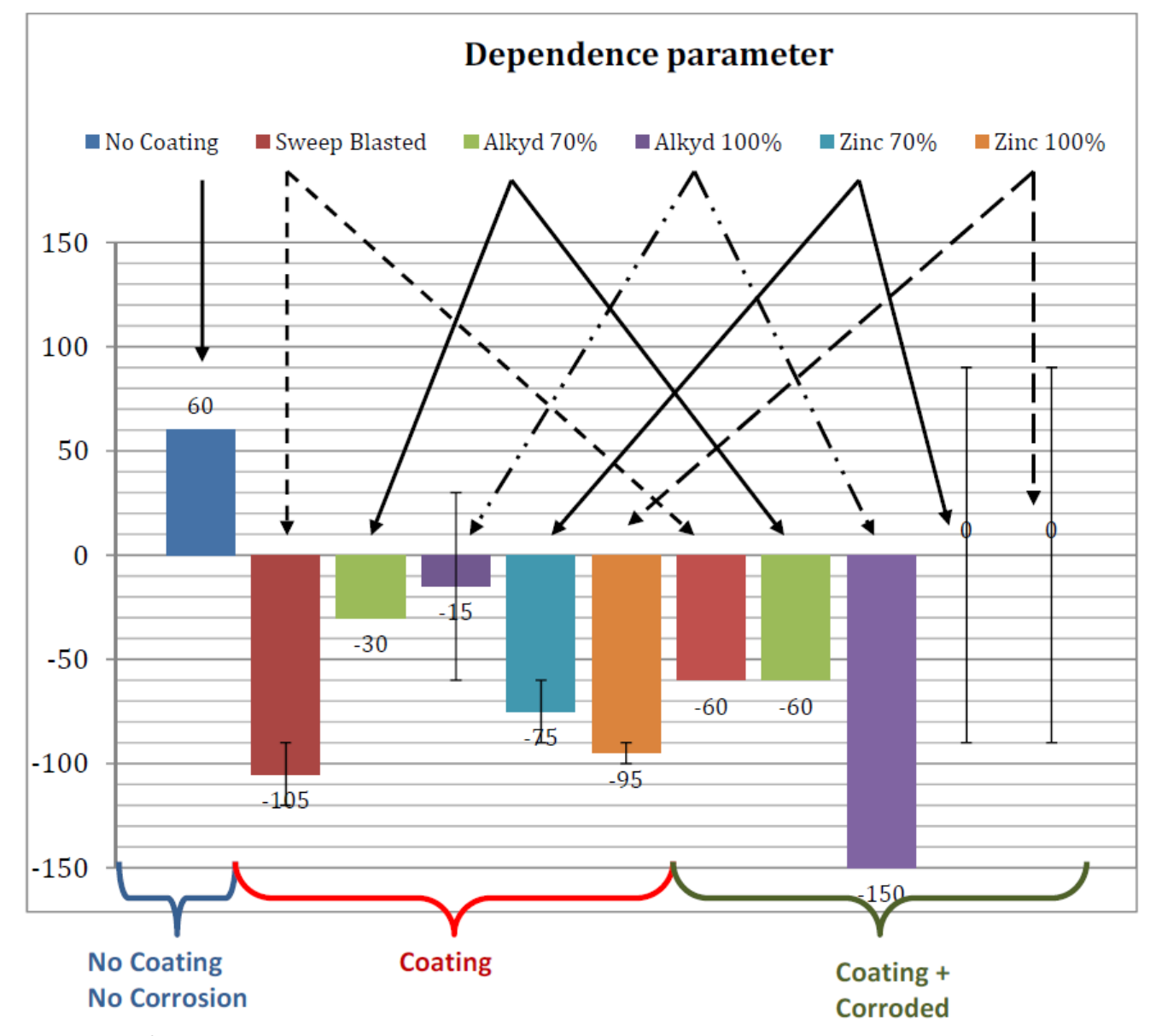

Figure 9: Impact of the corrosion and the corrosion-coating process on the velocity parameter. 


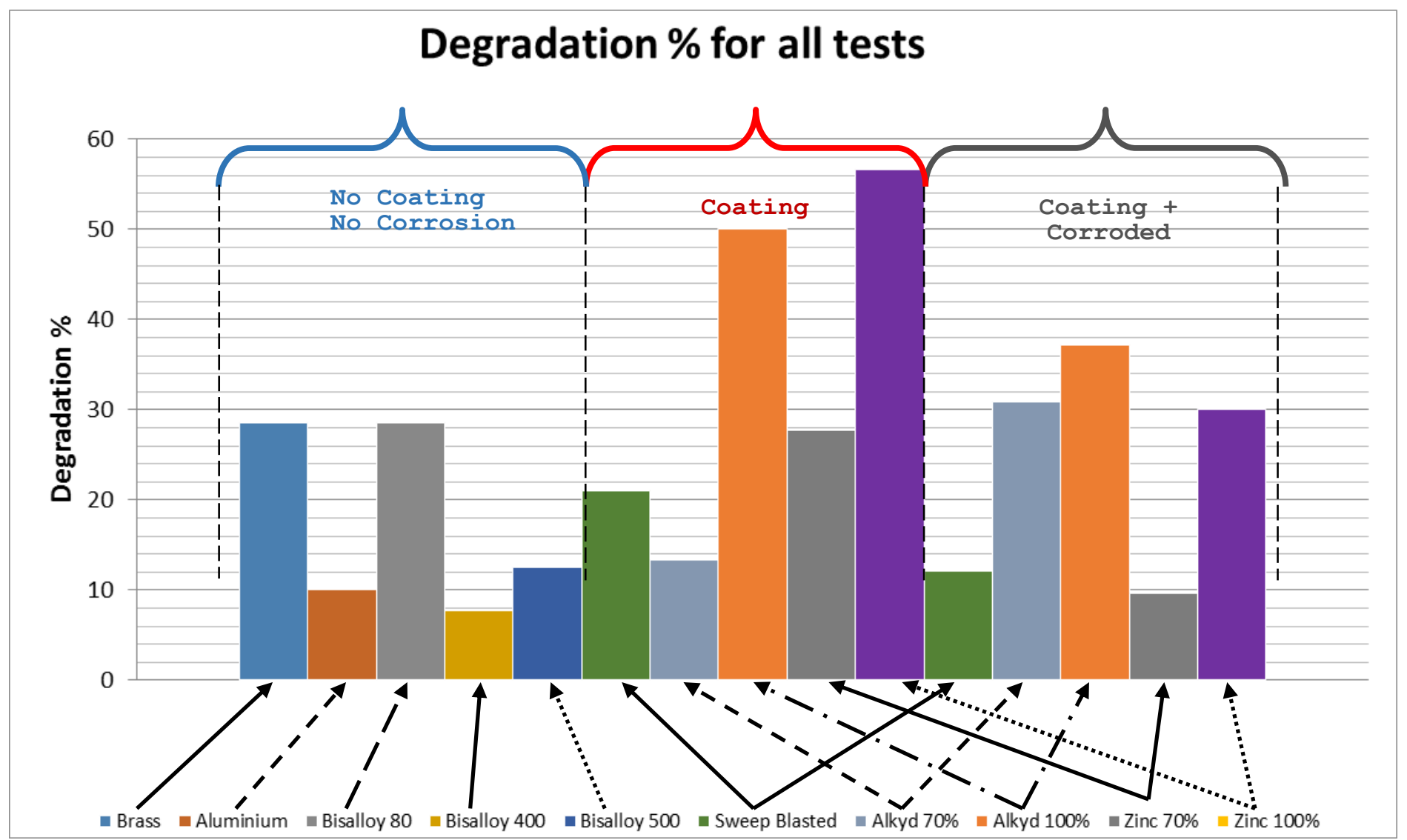

Figure 10: Degradation of the sliding force between the 1st and 2nd run for all cases 

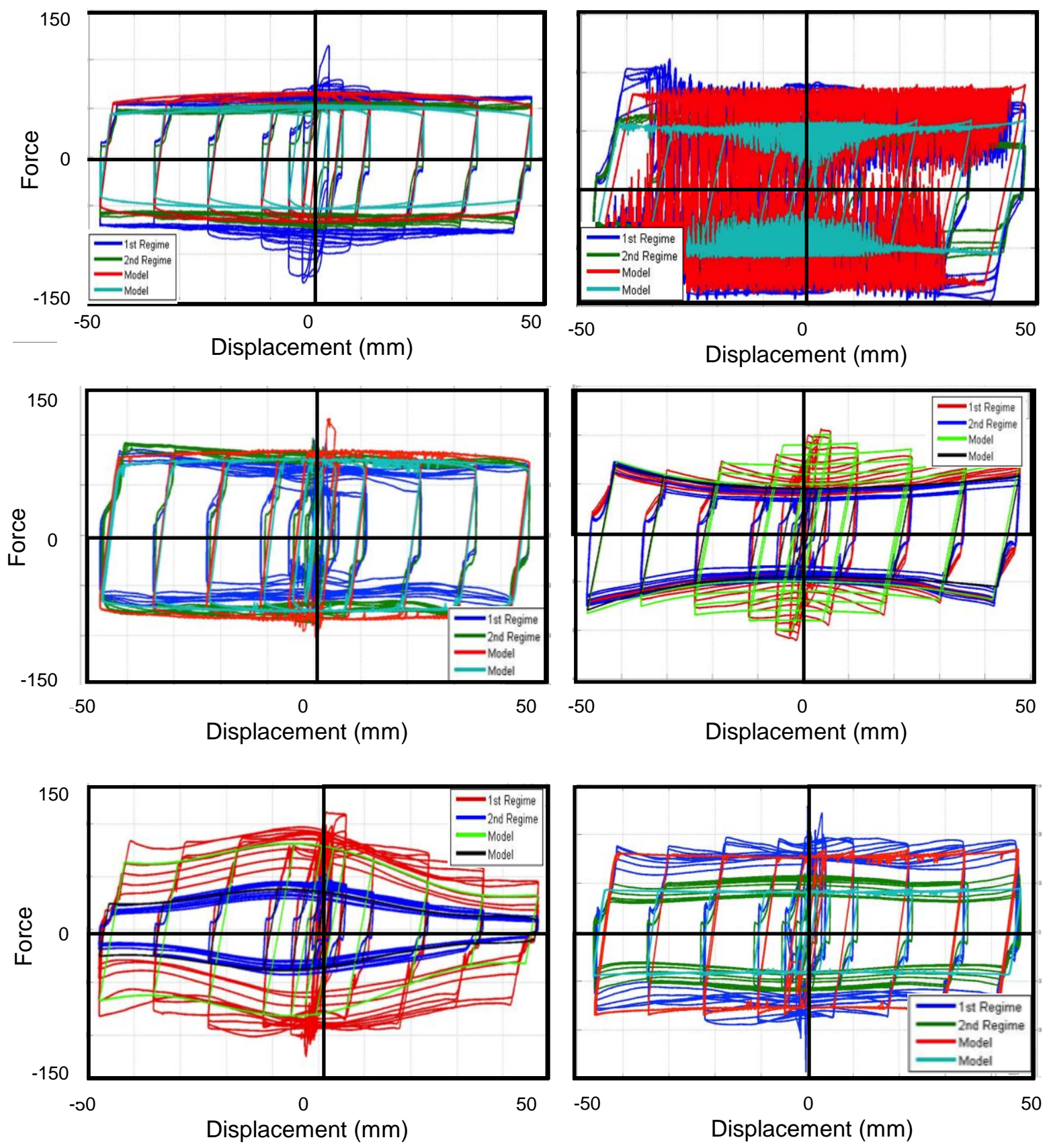

Figure 11: Range of experimental results for qualitative comparison of the wide range of possible hysteresis loops and levels of positive (and negative, see middle right panel) velocity dependence. Aluminium shim materials (top left), Zinc100\% control coating (top right), Zinc 70\% corrosion coating (middle left), Alkyd 70\% control coating (middle right), Alkyd 100\% control coating (bottom left) and Alkyd 100\% corrosion coating (bottom right) 


\subsection{Conclusions}

This research presents a model-based analysis of a series of experimental results. The model is used to quantify and characterise the mechanics of a range of AFC devices with different shim materials and anticorrosion coatings. This analysis thus provides a unique set of results, quantified by the model to guide design and use of these devices.

More specifically, this work presents a range of experimental results and an augmented, nonlinear Menegotto-Pinto model to capture the observed dynamics in a model suitable for design prediction. The overall results show that this model is suitable for approximating the performance of a wide range of Asymmetric Friction Connections (AFCs). It is also readily generalisable and any device of this type that exhibits consistent, similar hysteretic behaviour could be simulated with this type of elasto-plastic MenegottoPinto model, including the unique addition of velocity dependence. It thus shows promise for the increasing range of friction dissipation devices being developed and tested, as well as for better accounting for the observed variability in design.

More specifically, the addition of velocity dependence into the Menegotto-Pinto model improves its ability to replicate experimental results. All experimental results are consistent with the presence of velocity dependence, which is a novel finding for Coulomb friction devices, not previously though to exhibit such velocity dependence. At this time, the only conclusion about this dependence is that the anti-corrosion coating process generally leads to a negative velocity dependence parameter, implying a lubrication-like effect under cyclic input motions.

In addition, the range of materials and coatings tested indicate that there can be significant variability between devices. This variability ranges from positive to negative velocity coefficients in the model to very different performance for the same device with different anti-corrosion coatings. The variability in force and behaviour are significant and should be accounted for in design using models, such as the one presented, that can capture this range of variability within a fixed fundamental mechanics model.

Finally, the results corroborate the low-damage of these devices for large motion inputs. Degradation of most SHJ connections was approximately $20-30 \%$ over successive input motion test regimes, but was up to $60 \%$ 
for some anti-corrosion coatings. This degradation will limit the ability of the $\mathrm{SHJ}$ to dissipate energy and limit structural response on successive seismic events, such as those seen in the Christchurch series of earthquakes, without intervening inspection and repair. Therefore, this degradation should be taken into account be designers, as the SHJ may provide less resistive force during major aftershocks following a primary earthquake. 


\subsection{References}

1. Golondrino, J., et al., Design Considerations for the Braced Frames with Asymetric Friction Connections, in STESSA 2012 - Behaviour of Steel Structures in Seismic Areas. 2012: Santiago, Chile. p. 8-pages.

2. Clifton, C., Semi-Rigid Joints for Moments Resisting Steel Framed Seismic Resisting Systems, in Civil Engineering. 2005, Auckland: Auckland.

3. Khoo, H.H., et al., Development of the self-centering Sliding Hinge Joint with friction ring springs. Journal of Constructional Steel Research, 2012. 78: p. 201-211.

4. MacRae, G.A., et al., The Sliding Hinge Joint Moment Connection. Bulletin of the New Zealand Society for Earthquake Engineering, 2010. 43(3): p. 202-212.

5. Golondrino, J., et al., Behaviour of Asymmetrical Friction Connections using different shim materials, in New Zealand Society for Earthquake Enginering - Annual Conference 2012. 2012: Christchurch, New Zealand. p. 10-pages.

6. Rodgers, G.W., et al., Experimental development, tradeoff analysis and design implementation of high force-to-volume damping technology. Bulletin of the New Zealand Society for Earthquake Engineering, 2007. 40(2): p. 35-48.

7. Rodgers, G., et al., Performance Of A Damage-Protected Beam-Column Subassembly Utilizing External HF2V Energy Dissipation Devices. Earthquake Engineering \& Structural Dynamics (EESD), 2008. 37(3): p. 1549-1564.

8. Chase, J.G., et al., Re-shaping hysteretic behaviour using semi-active resettable device dampers. Engineering Structures, 2006. 28(10): p. 1418-1429.

9. Mulligan, K.J., et al., Experimental validation of semi-active resetable actuators in a 1/5th scale test structure. Earthquake Engineering \& Structural Dynamics, 2009. 38(4): p. 517-536.

10. Pekcan, G., J.B. Mander, and S.S. Chen, Fundamental Considerations for The Design of Non-linear Viscous Dampers. Earthquake Engineering and Structural Dynamics, 1999. 28: p. 1405-1425.

11. Dyke, S.J. and B.F. Spencer, Modeling and control of magnetorheological dampers for seismic response reduction. Smart Materials and Structures, 1996. 5: p. 565-575.

12. Jabbari, F. and J.E. Bobrow, Vibration Suppression with a Resetable Device. ASCE Journal of Engineering Mechanics, 2002. 128(9): p. 916-924.

13. Menegotto, M. and P. Pinto, Method of analysis for cyclically loaded reinforced concrete plane frames including changes in geometry and non-elastic behavior of elements under combined normal force and bending, in Proc. Symp. Resistance and Ultimate Deformability of Struct. Acted on by WellDefined Repeated Loads, IABSE Reports, Volume 13. 1973, IABSE Reports: Lisbon, Portugal.

14. Borzouie, J., et al., Experimental studies on cyclic performance of column base weak axis aligned asymmetric friction connection. Journal of Constructional Steel Research, 2015. 112: p. 252-262.

15. Borzouie, J., et al., Experimental Studies on Cyclic Performance of Column Base Strong Axis-Aligned Asymmetric Friction Connections. Journal of Structural Engineering, 2016. 142(1).

16. Herve, R., et al., Dynamic Friction Co-efficient of Asymmetrical Friction Connections (AFC), in New Zealand Society of Earthquake Engineers Annual Conference (NZSEE 2013). 2013: Wellington, New Zealand. p. 8-pages. 